\title{
A FURTHER NOTE ON CERTAIN INTEGRAL EQUATIONS OF ABEL TYPE
}

\section{by J. BURLAK \\ (Received 28th February 1964)}

In a recent note (1) Srivastav considered the solution of the following integral equations of Abel type

$$
\begin{aligned}
& \int_{a}^{x}\{f(x)-f(t)\}^{-\alpha} h(t) d t=g(x), \quad a<x<b, \quad 0<\alpha<1, \\
& \int_{x}^{b}\{f(t)-f(x)\}^{-\alpha} h(t) d t=g(x), \quad a<x<b, \quad 0<\alpha<1,
\end{aligned}
$$

where $f(x)$ is monotonic strictly increasing and differentiable for $a \leqq x \leqq b$ and $f^{\prime}(x) \neq 0$ in this interval. Only the first of these conditions was stated in (1) but the second is necessary for the form of solution given in (1) to be valid and the third is necessary for the integrals in (1) and (2) to exist at all. For, if $f^{\prime}\left(x_{0}\right)=0, a<x_{0}<b$, then

$$
f(t)-f\left(x_{0}\right)=o\left(t-x_{0}\right)=O\left(\left(t-x_{0}\right)^{1+\delta}\right), \quad \delta \geqslant 0,
$$

where, for example, $\delta>0$ whenever $f^{\prime \prime}(x)$ exists for $a<x<b$, and for $x=x_{0}$ the integrals will only exist under the more stringent condition on $\alpha: 0<\alpha(1+\delta)<1$.

The purpose of this further note is to point out that the integral equations (1) and (2) are in fact no more general than the original Abel equation (i.e. than equation (1) with $f(x)=x$ ).

Thus, if we put $f(x)=\xi, f(t)=\tau, f(a)=\beta, f(b)=\gamma, h(t) / f^{\prime}(t)=H(\tau)$, $g(x)=G(\xi)$, we see that (1) reduces to

$$
\int_{\beta}^{\xi}(\xi-\tau)^{-\alpha} H(\tau) d \tau=G(\xi), \quad \beta<\xi<\gamma, \quad 0<\alpha<1,
$$

where the conditions on $f(x)$ ensure both the existence of the inverse function $x=f^{-1}(\xi)$ and the fact that $\beta<\tau<\gamma$. But (3) is just Abel's equation with solution (2)

$$
H(\tau)=\frac{\sin \alpha \pi}{\pi} \frac{d}{d \tau} \int_{\beta}^{\tau}(\tau-\eta)^{\alpha-1} G(\eta) d \eta
$$

whence we immediately deduce that

$$
h(t)=\frac{\sin \alpha \pi}{\pi} \frac{d}{d t} \int_{a}^{t}\{f(t)-f(u)\}^{\alpha-1} g(u) f^{\prime}(u) d u .
$$


The same substitution reduces (2) to

$$
\int_{\xi}^{\gamma}(\tau-\xi)^{-\alpha} H(\tau) d \tau=G(\xi), \quad \beta<\xi<\gamma, \quad 0<\alpha<1 .
$$

But now a further elementary substitution reduces (5) to (3) and so we obtain

and this yields

$$
H(\tau)=-\frac{\sin \alpha \pi}{\pi} \frac{d}{d \tau} \int_{\tau}^{\gamma}(\eta-\tau)^{\alpha-1} G(\eta) d \eta
$$

$$
h(t)=-\frac{\sin \alpha \pi}{\pi} \frac{d}{d t} \int_{t}^{b}\{f(u)-f(t)\}^{\alpha-1} g(u) f^{\prime}(u) d u .
$$

The solutions (4) and (6) are those given in (1)

\section{REFERENCES}

(1) R. P. Srivastav, Proc. Edinburgh Math. Soc., 13 (1963), 271-272.

(2) M. BôCHER, An Introduction to the Study of Integral Equations (Cambridge, 1909), p. 8.

\section{Mathematics Department}

THE UNIVERSITY

Glasgow, W.2 